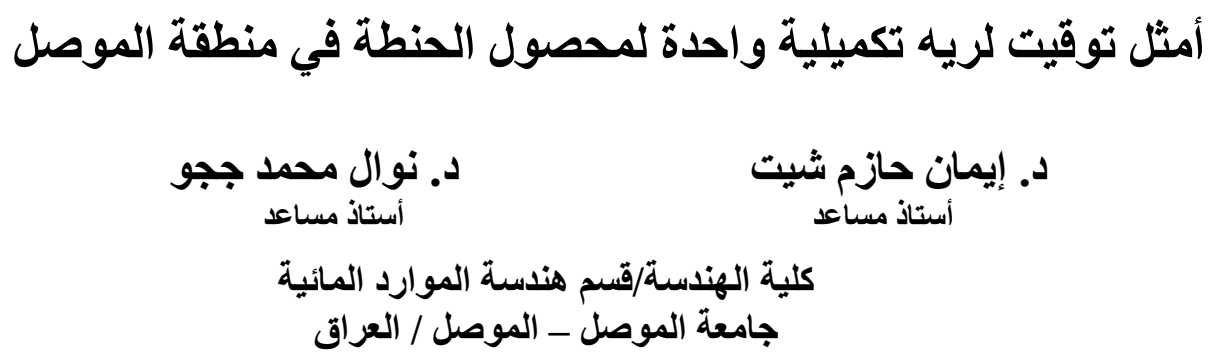

\title{
الخلاصة
}

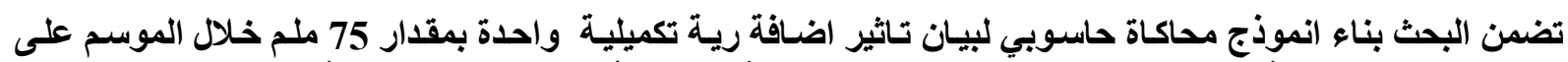

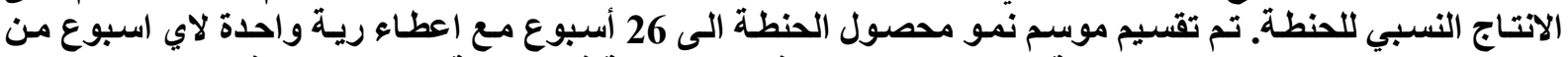

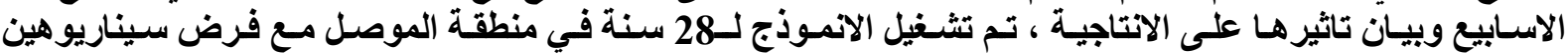

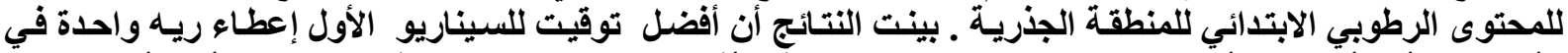

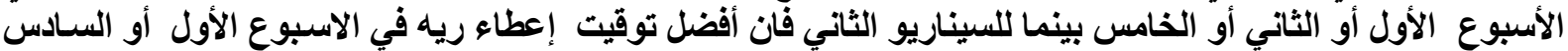

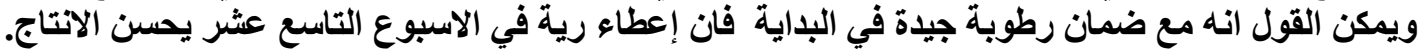

\section{Optimal Timing of One Supplemental Irrigation}

\author{
Dr. Eman Hazim Sheet Dr. Nawal M . Jajjo \\ Assistant Professor Assistant Professor \\ Water Resources Department, \\ College of Engineering, University of Mosul , Mosul, Iraq
}

\begin{abstract}
A computer simulation model is developed to study the effect of adding one supplemental irrigation of $75 \mathrm{~mm}$ on the relative yield of wheat . The growing season of wheat is divided into 26 weeks and a single irrigation is added to any one of the weeks . The model is run for 28 years through the growing season of wheat with two different scenarios for initial water contents in the root zone. The results show that for the first scenario the best single supplemental irrigation is at the first or second or the fifth week, but for the second scenario is at the first or sixth week, it can be said that with good initial moisture in the root zone at the beginning the best choice is to add single irrigation at the $19^{\text {th }}$ week.
\end{abstract}




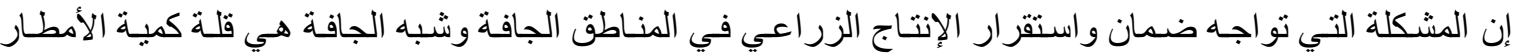

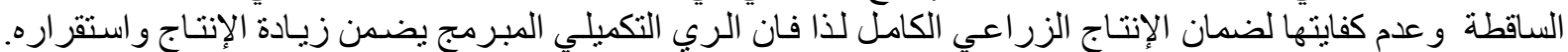

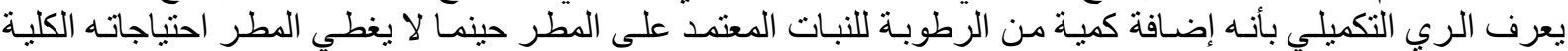

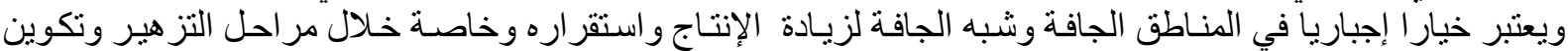

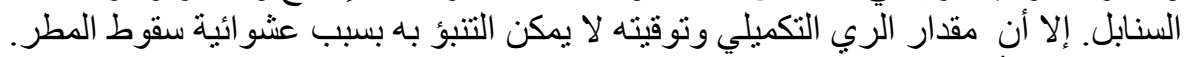

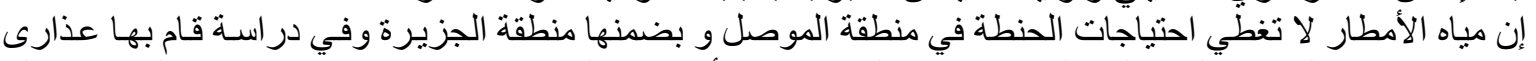

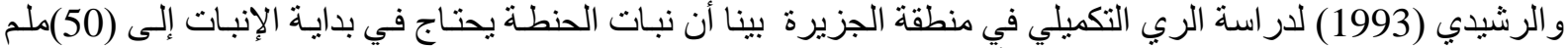

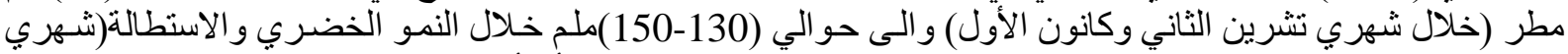

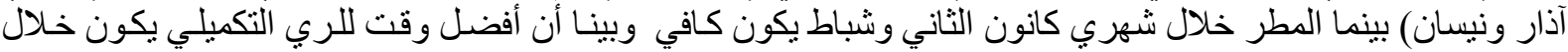

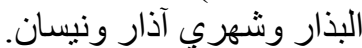

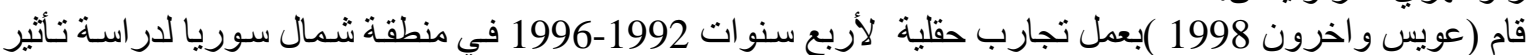

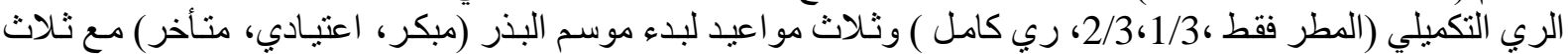

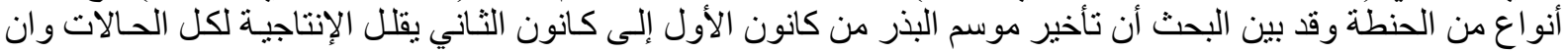

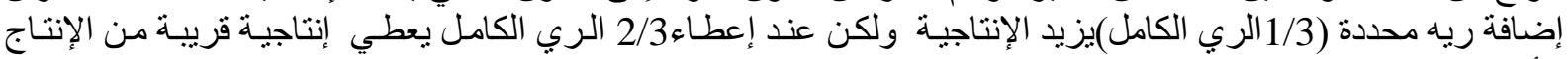

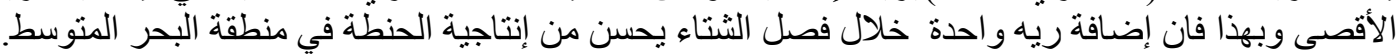

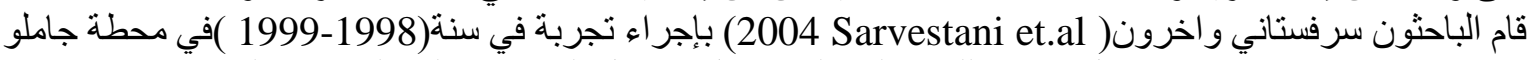

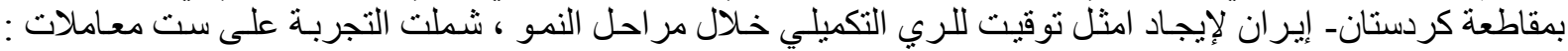

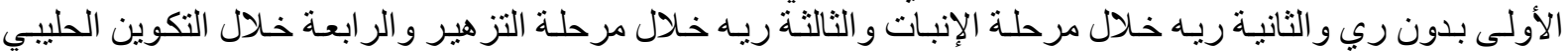
milking stage

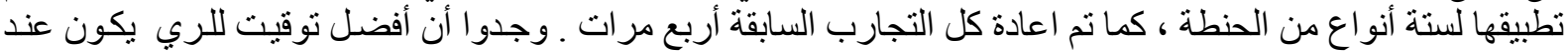

إعطاء ريتين عند الإنبات و التكوين الحليبي.

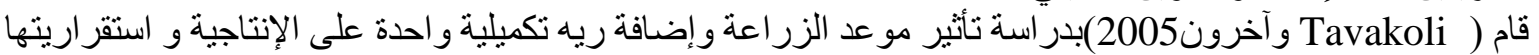

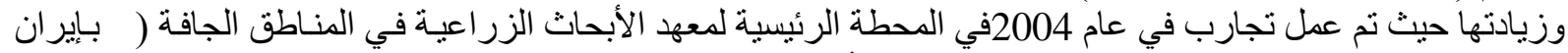

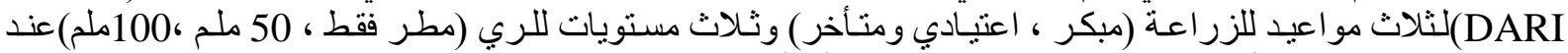

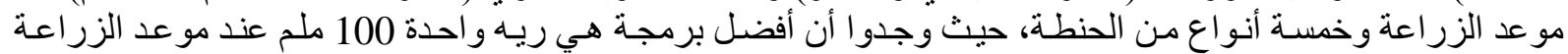

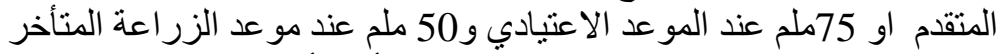

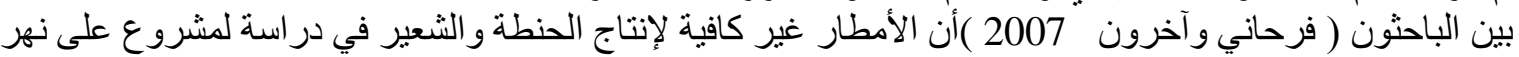

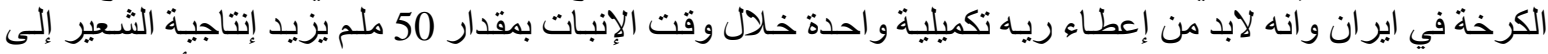

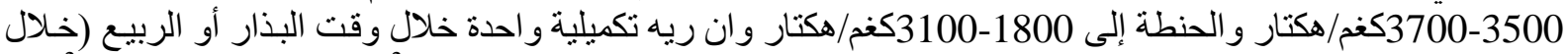

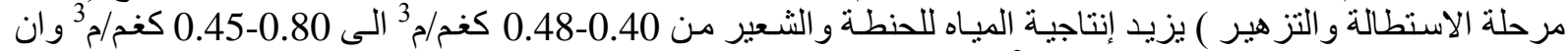

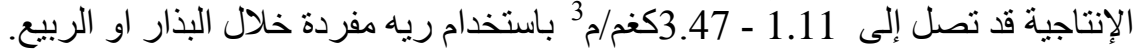

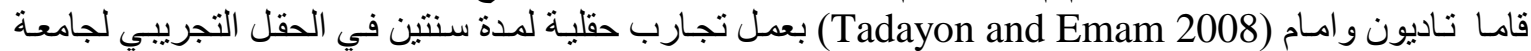

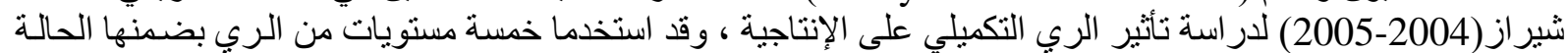

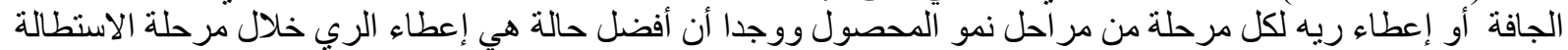

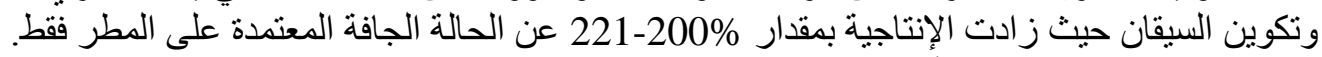

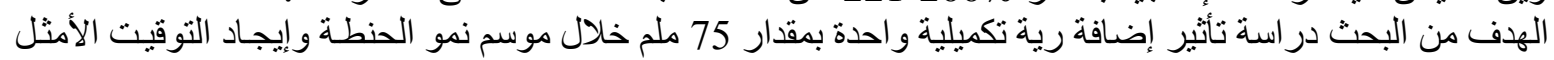

$$
\text { الذي يعطي أعلى إنتاجية. }
$$

\section{صياغة أنموذج المحاكاة}

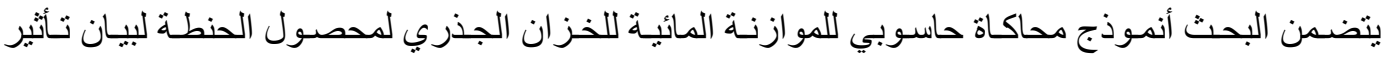

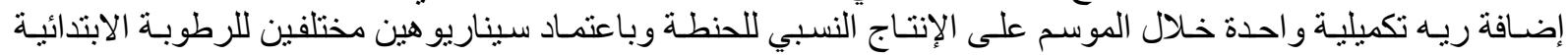
لمنطقة الجذرية.

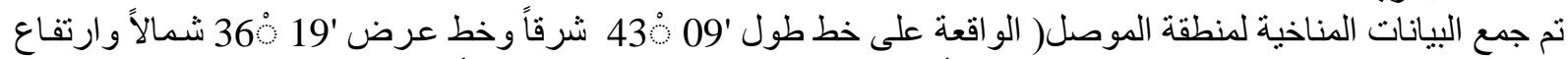

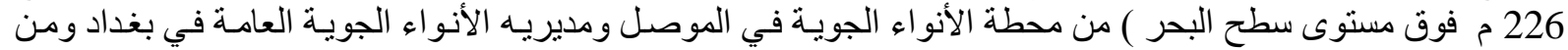

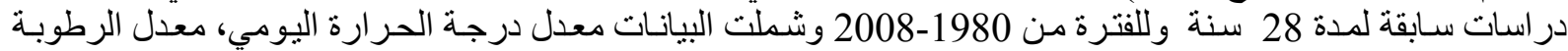
النسبية اليومي، سرعة الرياح على ارتفاع 2 م ، عدد ساعات الثروق و أعماق الأمطار اليومية . 
أفترضت الدراسة تربة متوسطة النسجة بسعة حقلية 3.1 ملم /سم ونقطة ذبول 1.5 ملم /سم مع محتوى رطوبي ابتدائي

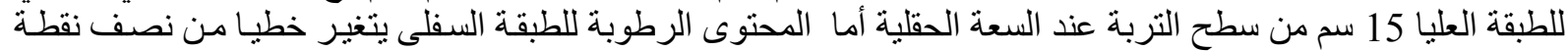

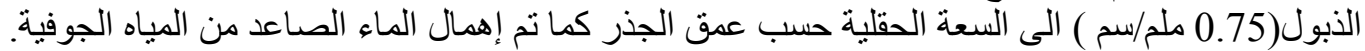

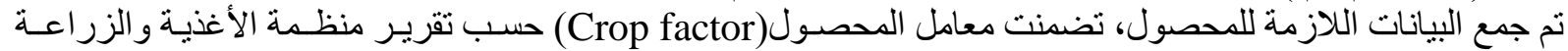

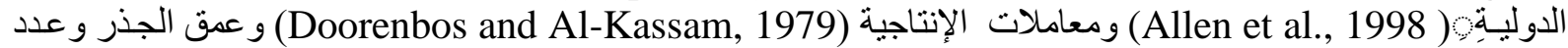

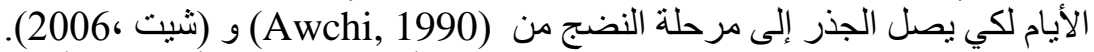

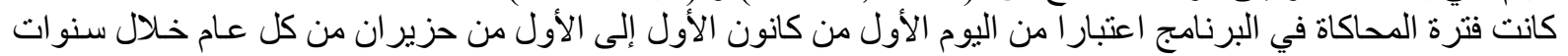

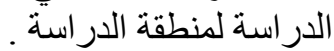

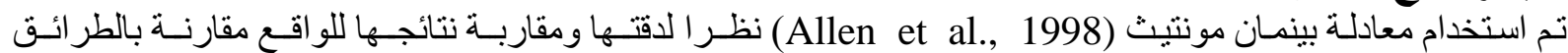
الأخرى. أن صيغة معادلة الفاو بينمان مونتيث هي:

$E T_{\circ}=\frac{0.408 \Delta\left(R_{n}-G\right)+\gamma \frac{900}{T+273} U_{2}(e s-e a)}{\Delta+\gamma\left(1+0.34 U_{2}\right)}$

حيث أنّ: ET。

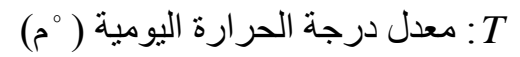

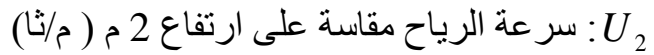

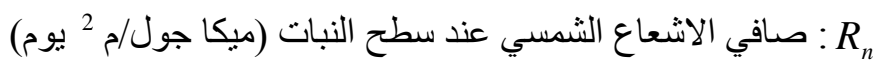

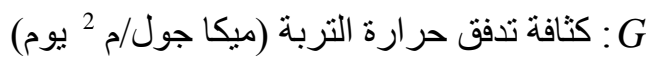

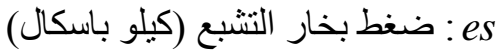
ea (النص في ضغط بخار التشبع (كيلو باسكال) : es -ea

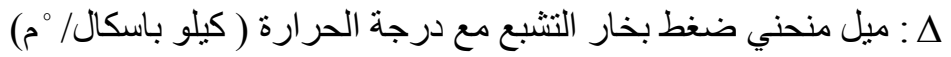

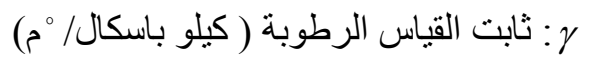

التبخر-النتح للمحصول( Crop evapotranspiration): تم حساب التبخر -النتح للمصصول من حاصل ضرب التبخر -النتح الكامن في معامل المحصول:

$C P E T=E T_{\circ} * K_{c}$

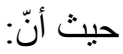

$$
\begin{aligned}
& \text { CPET } \\
& \text { ETo } \\
& \text { K }
\end{aligned}
$$

تم إيجاد معاملات المحصول(crop coefficient) اليوميـة باعنماد التقريب البيني (interpolation) لمعاملات

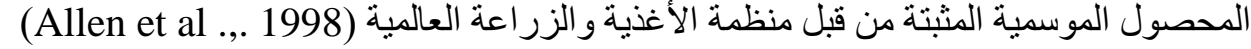

التبخر-النتح الحقيقي (Actual evapotranspiration):

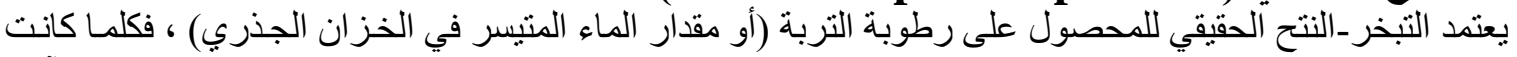

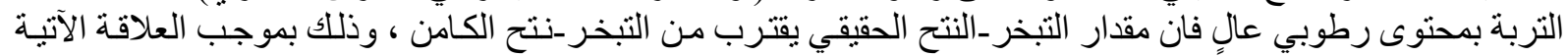
: (Allen et al . 1998)

$$
E T a=C P E T * K s
$$




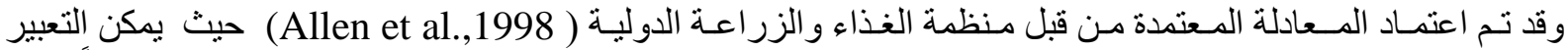

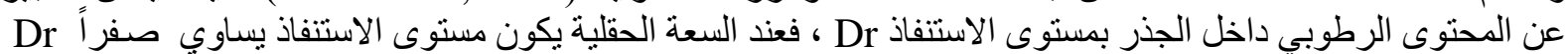

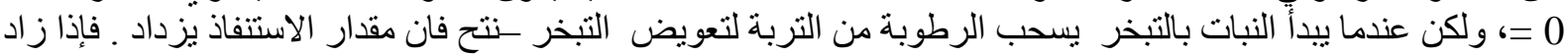

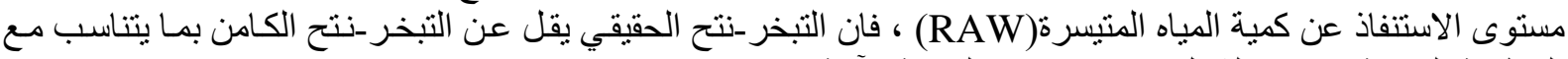

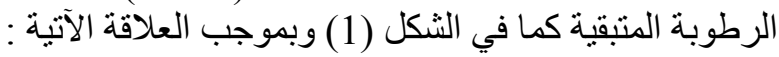

$$
K s=\frac{T A W-D r}{(1-p) T A W}
$$

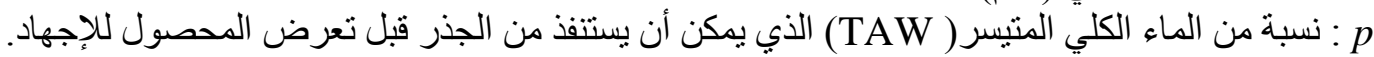

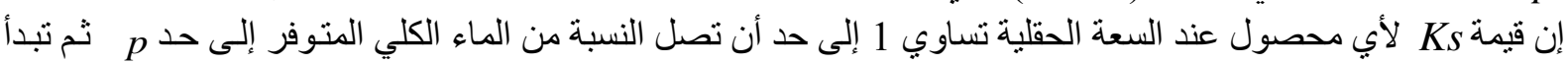

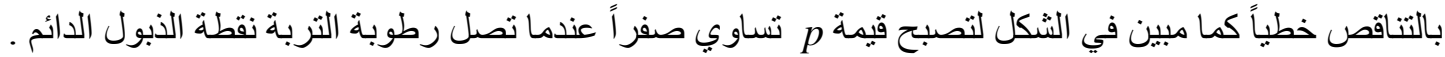

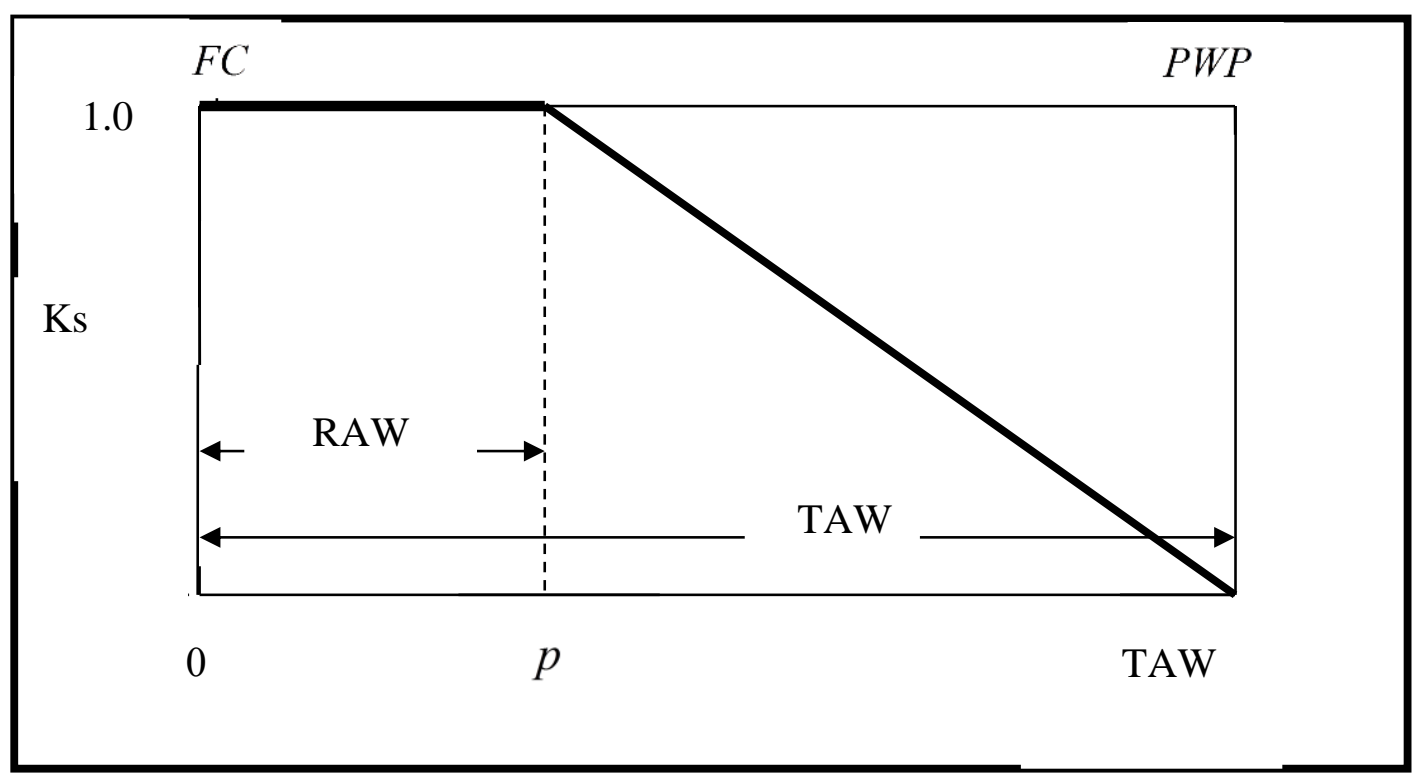

الثكل (1) معامل جاهزية الرطوبة كدالة للماء المتيسر في التربة (Allen et al., 1998).

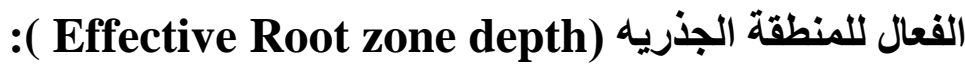

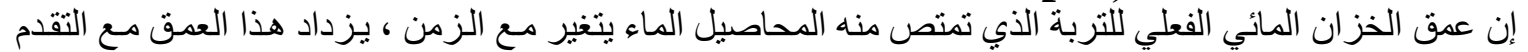

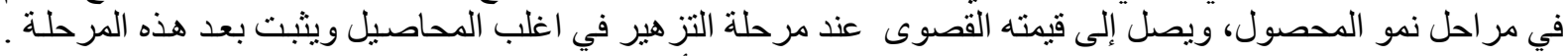

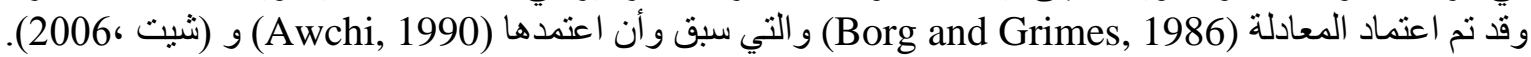

$$
R Z D(I)=M R D\left[0.5+0.5 \sin \left\{3.03\left(\frac{I}{D m}\right)-1.47\right\}\right]
$$

$$
\text { I : تسلسل اليوم في فترة نمو المحصول . I }
$$




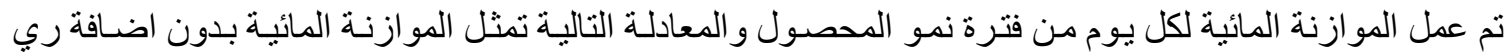

$9 \quad A W E=A W B+R A I N-E T a-D R A I N$

حيث أنّ:

AWE

AWB

RAIN

DRAIN

أما المو ازنة المائية اليومية للمحصول مع إضافة ري تكميلي (IR ) بالملم فتمثلها المعادلة التالية:

$$
A W E=A W B+R A I N-E T a-D R A I N+I R
$$

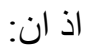

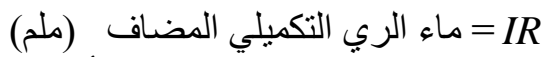
تم تقسيم موسم نمو المحصول إلى أربعة مر احل وإيجاد طول كل مرحلة مع معامل الإنتاجية كما في الجدول (1) لمأخوذة من (Doorenbos and Al-Kassam, 1979 ) وهي كالاتي : $Y_{i}=\frac{Y a}{Y m}=\prod_{j=1}^{n s}\left[1-k y_{j}\left(1-\left(\frac{E T a}{C P E T}\right)_{j}\right]\right.$

$$
\begin{aligned}
& \text { Ya } \\
& \text { : أقصى إنتاج للمحصول. : Ym }
\end{aligned}
$$

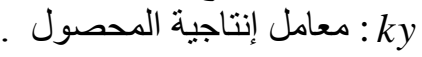

\begin{tabular}{|c|c|c|}
\hline معامل الانتاجية ky & طول المرحلة يو مِ & المرحلة \\
\hline 0.2 & 27 & الأولى : الابتدائية \\
\hline 0.6 & 81 & الثانية: النمو الخضري \\
\hline 0.5 & 38 & الثالثة: وسط الموسم \\
\hline 0.5 & 37 & الر ابعة: أواخر الموسم \\
\hline
\end{tabular}

$$
\begin{aligned}
& \text { j : تسلسل المرحلة } \\
& \text { ns }
\end{aligned}
$$

الجدول(1) : معاملات الإنتاجية لمر احل نمو الحنطة

تم فرض سيناريو هين مختلفين للمحتوى الرطوبة الابتدائي للمنطقة الجذرية، الأول تكون به الطبقة السطحية (بعمق 15سم

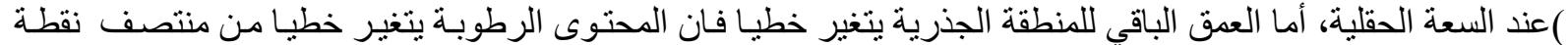

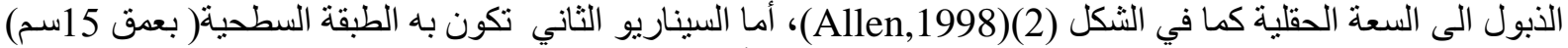

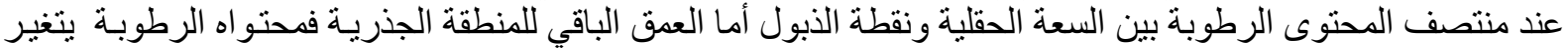

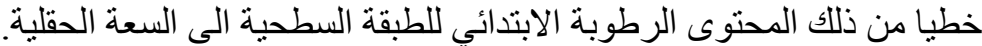
وقد تم إعطاء ريه تكميلية مقدارها 75 ملم لكل أسبو عن الأسابيع ألـ (26) و إيجاد الإنتاج النسبي لجميع السنوات. 
No. 3

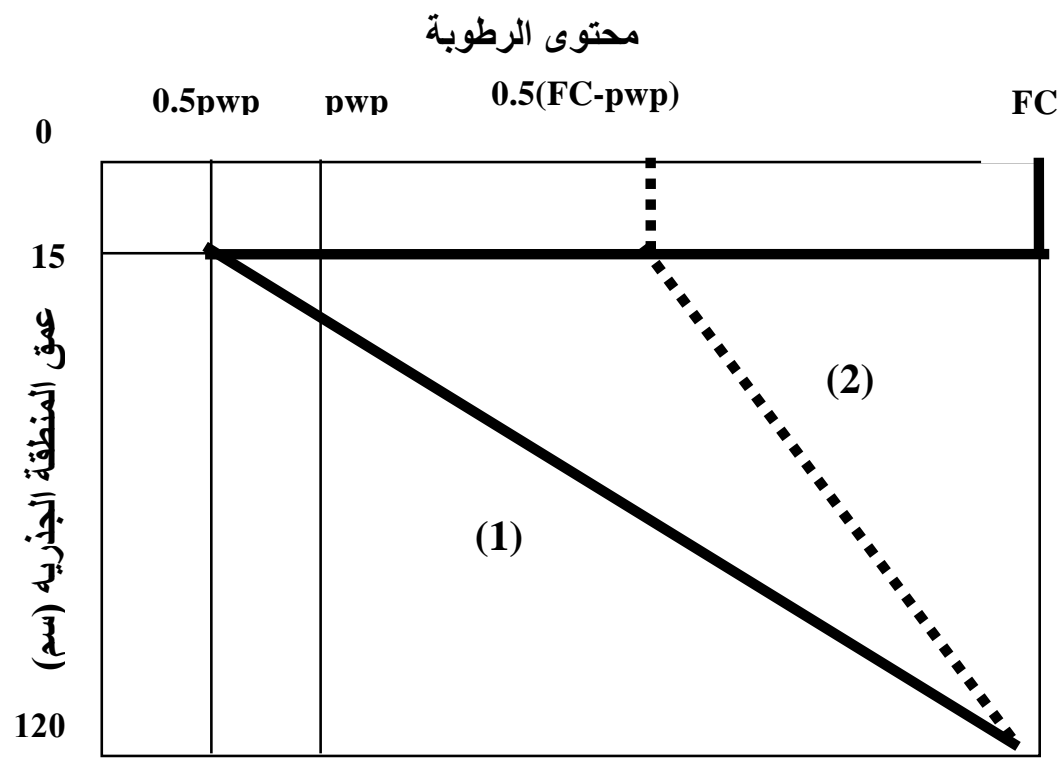

الثكل(2): توزيع محتوى الرطوبة الابتدائي في المنطقة الجذريه

\section{النتائج والمناقشُة}

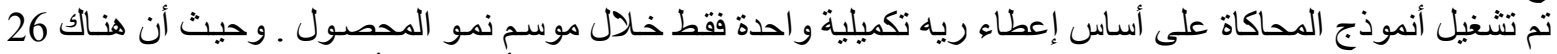

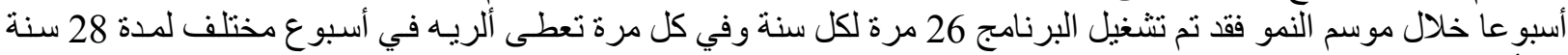

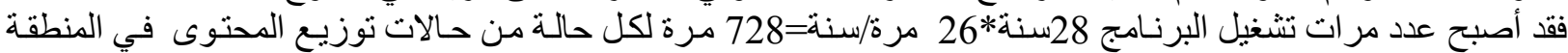

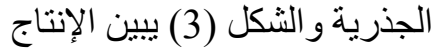
النسبي لبعض السنو ات عند إعطاء ريه لأي أسبوع من الأسابيع للسيناريو الأول للمحتوى الرطوبة.

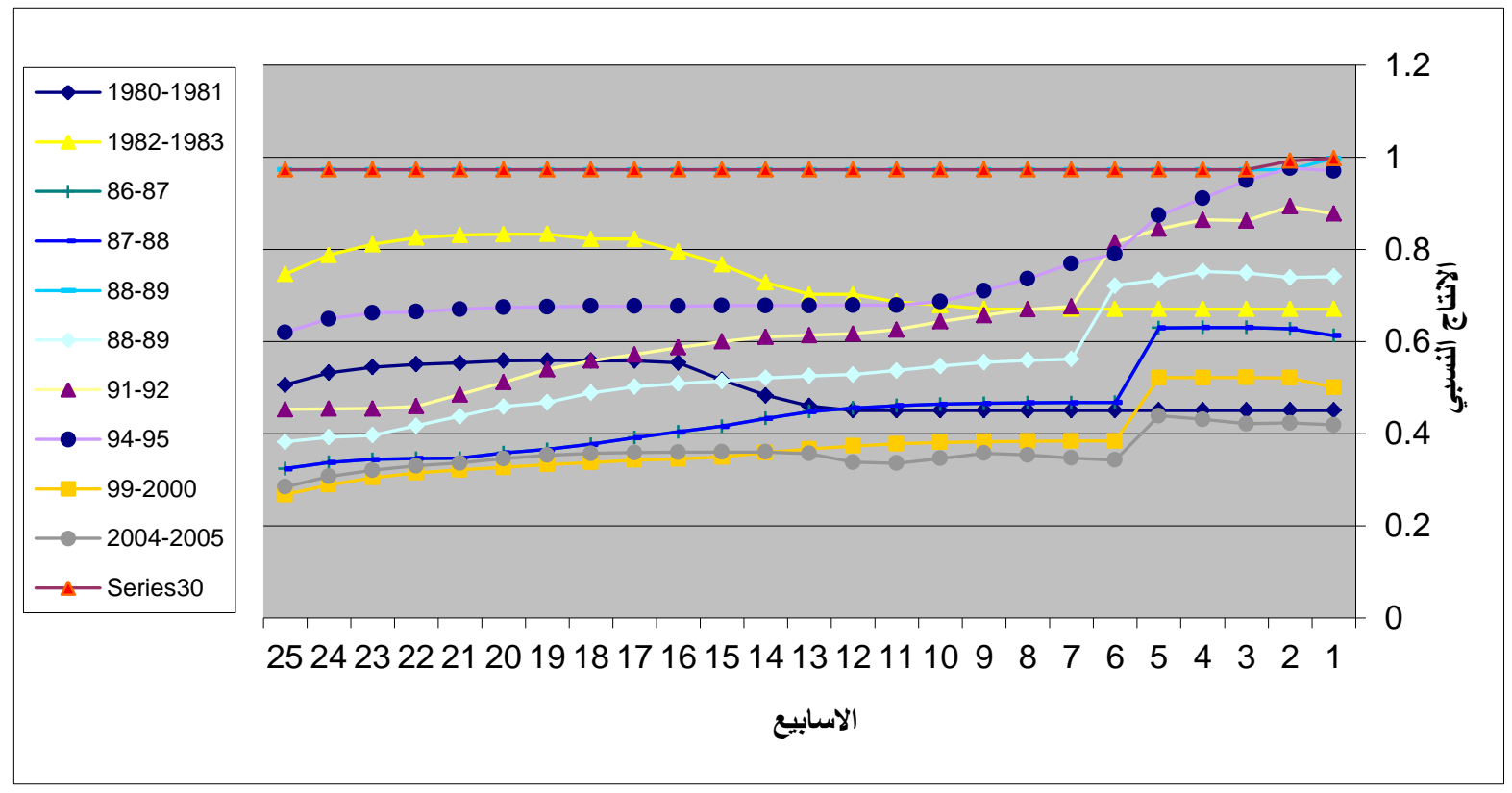

الثكل(3): الانتاج النسبي لبعض السنوات عند اعطاء ريه لأي اسبوع من الاسابيع للسيناريو الاول 


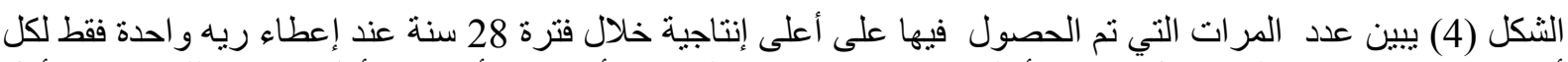

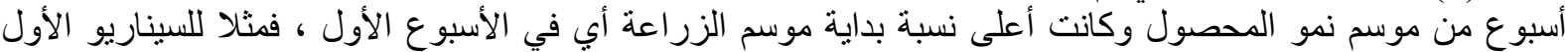

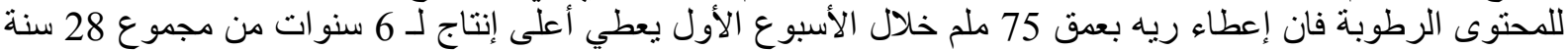

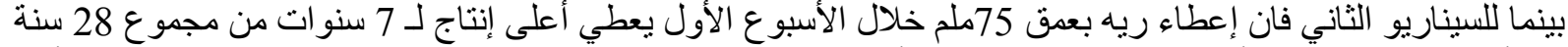
كما أن إعطاء ريه في الأسبوع التاسع عشر يعطي أعلى إنتاجية لثناث سنوات من مجموع 28سنة وللإنيناريو هين الأول

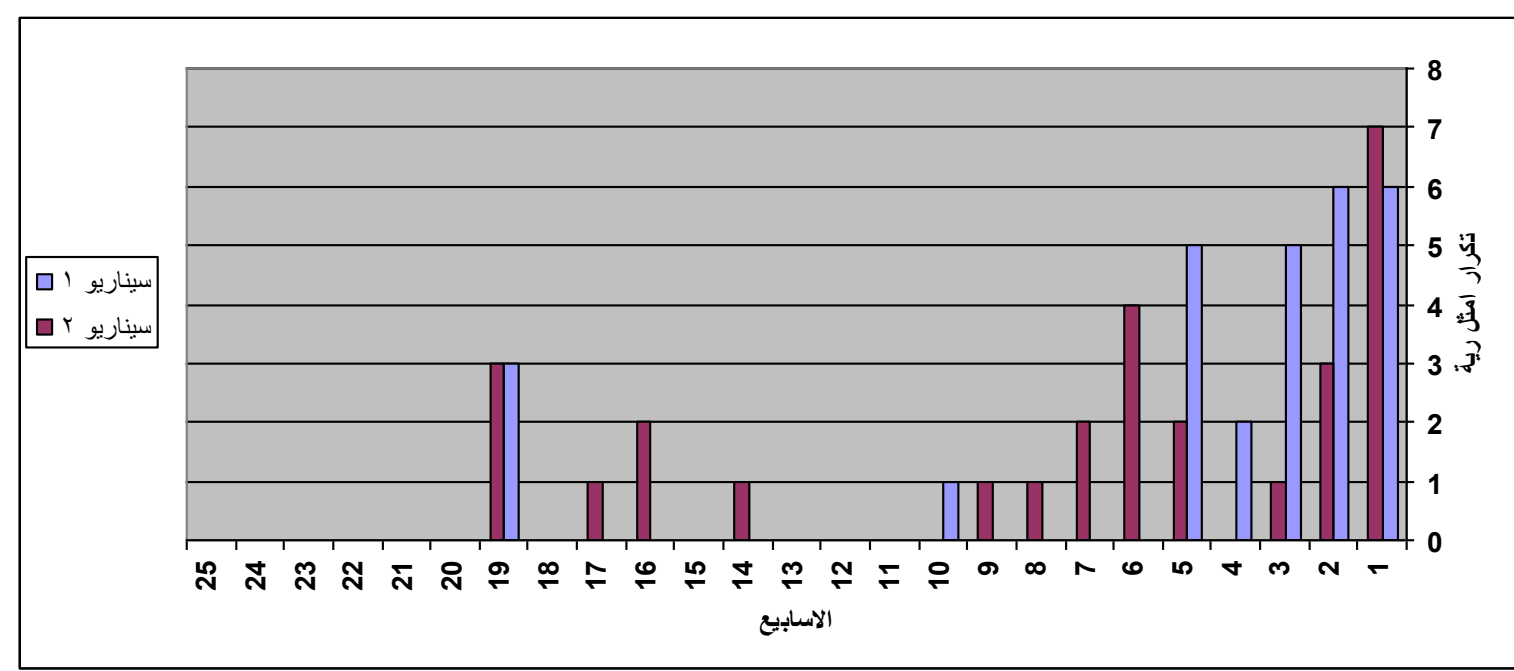

$$
\text { الثكل (4): عدد السنو ات التي حصلت على اعلى انتاج لكل اسبوع من موسم النمو }
$$

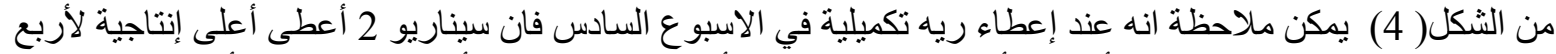

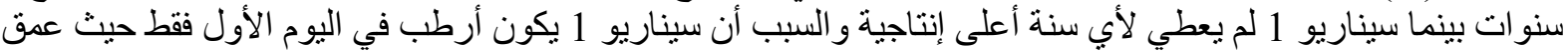

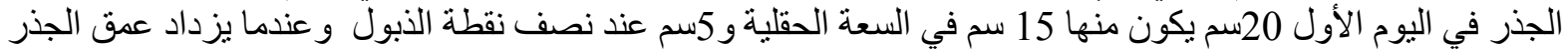

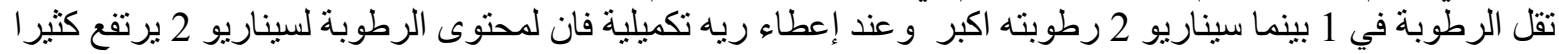

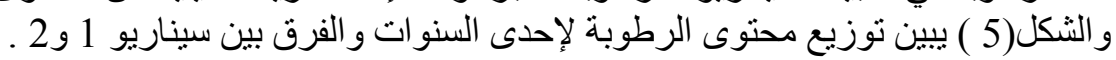

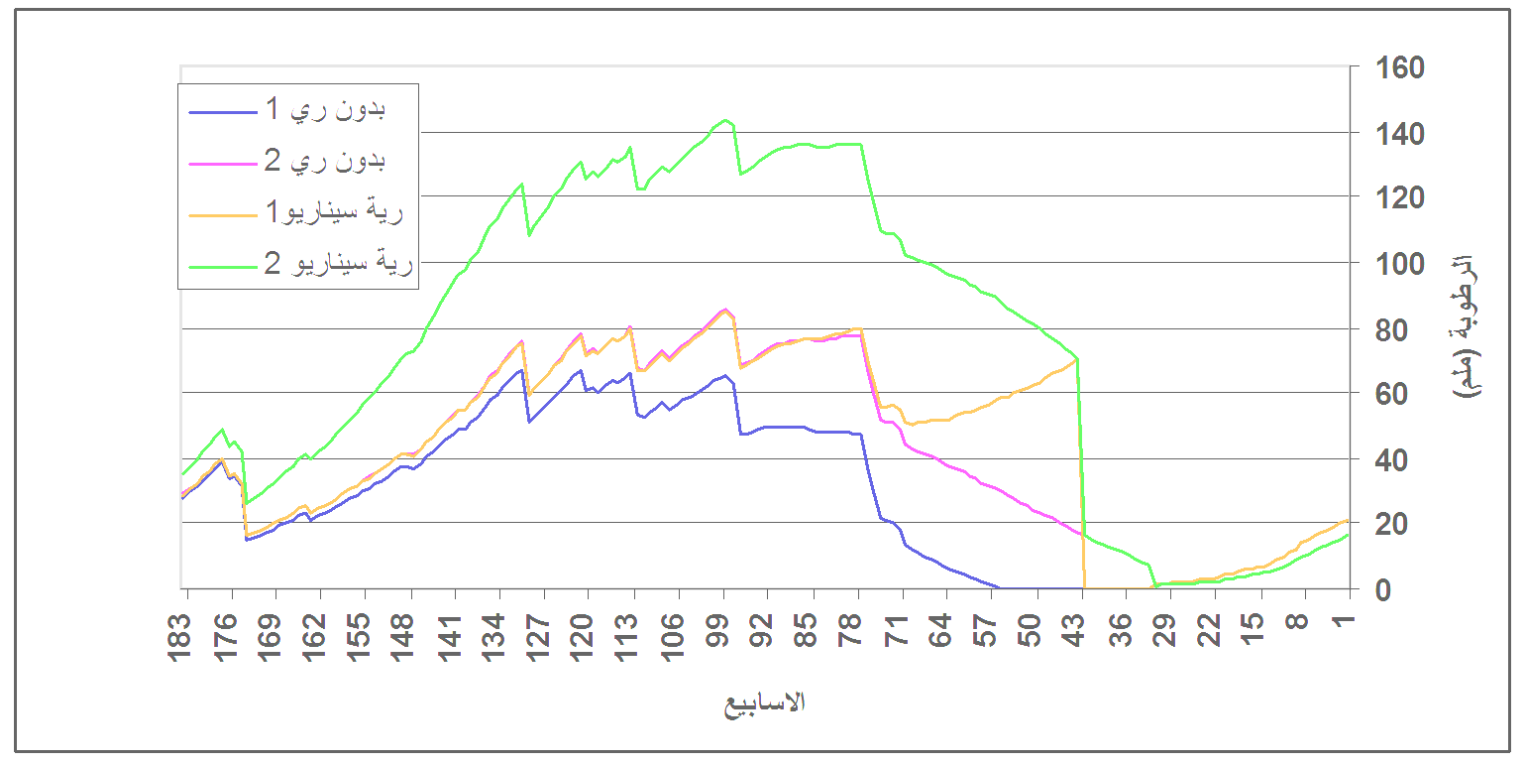

الثكل(5): توزيع الرطوبة لكل اسبوع من الاسابيع على طول موسم النمو لسنة 2000-2001 


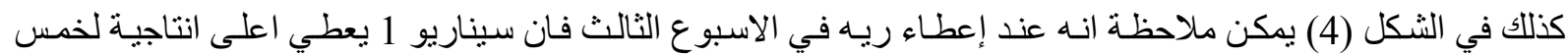

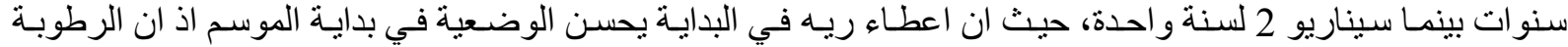

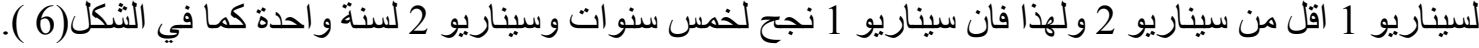

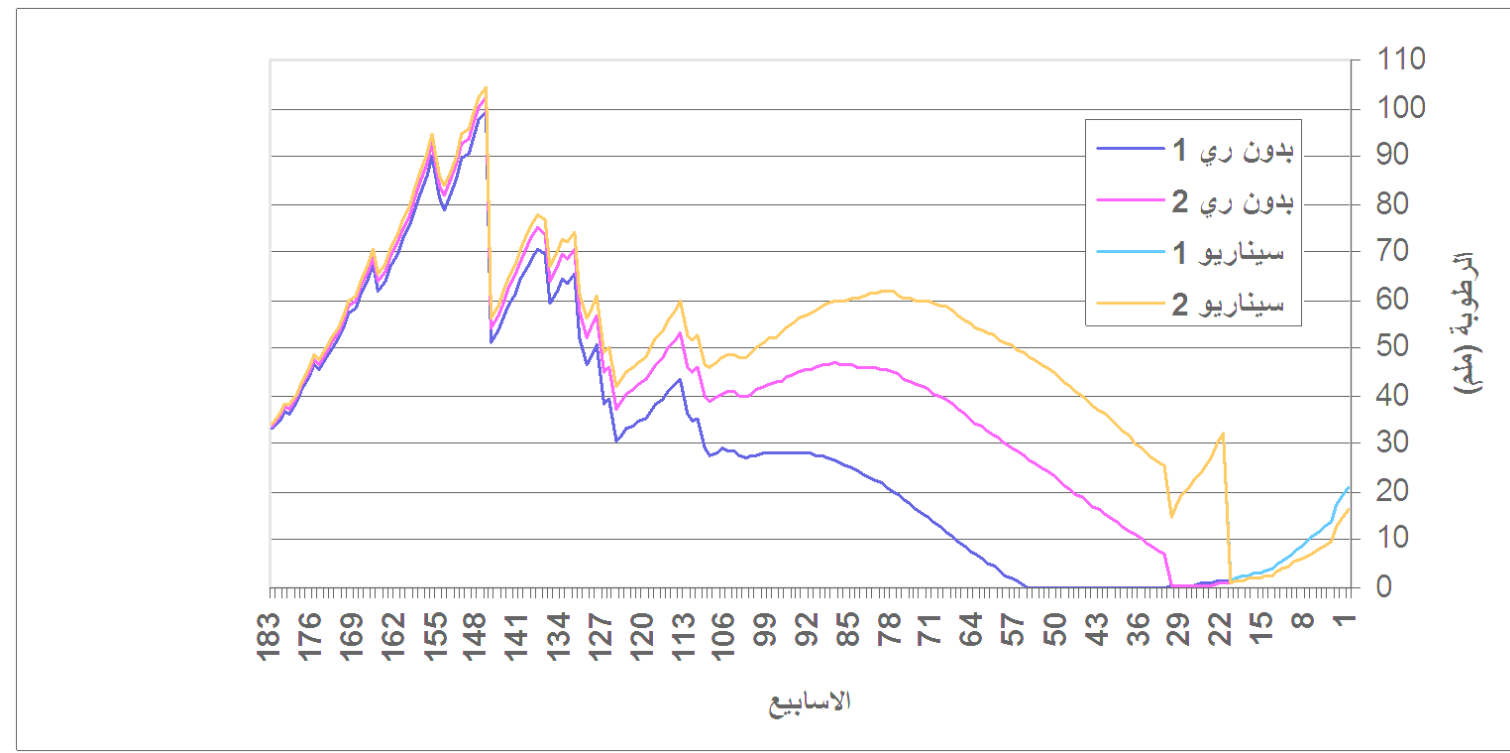

الثكل(6): توزيع الرطوبة لكل اسبوع من الاسابيع على طول موسم النمو لسنة 1985-1986

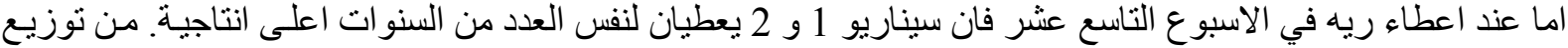

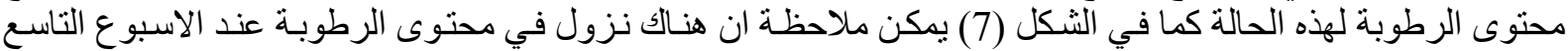

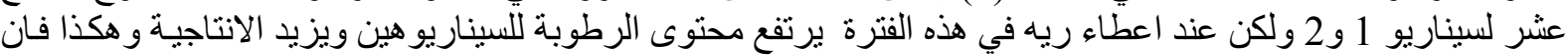
توقيت الري مع المطر ملائم في الاسبوع 19 لتنلك السنة

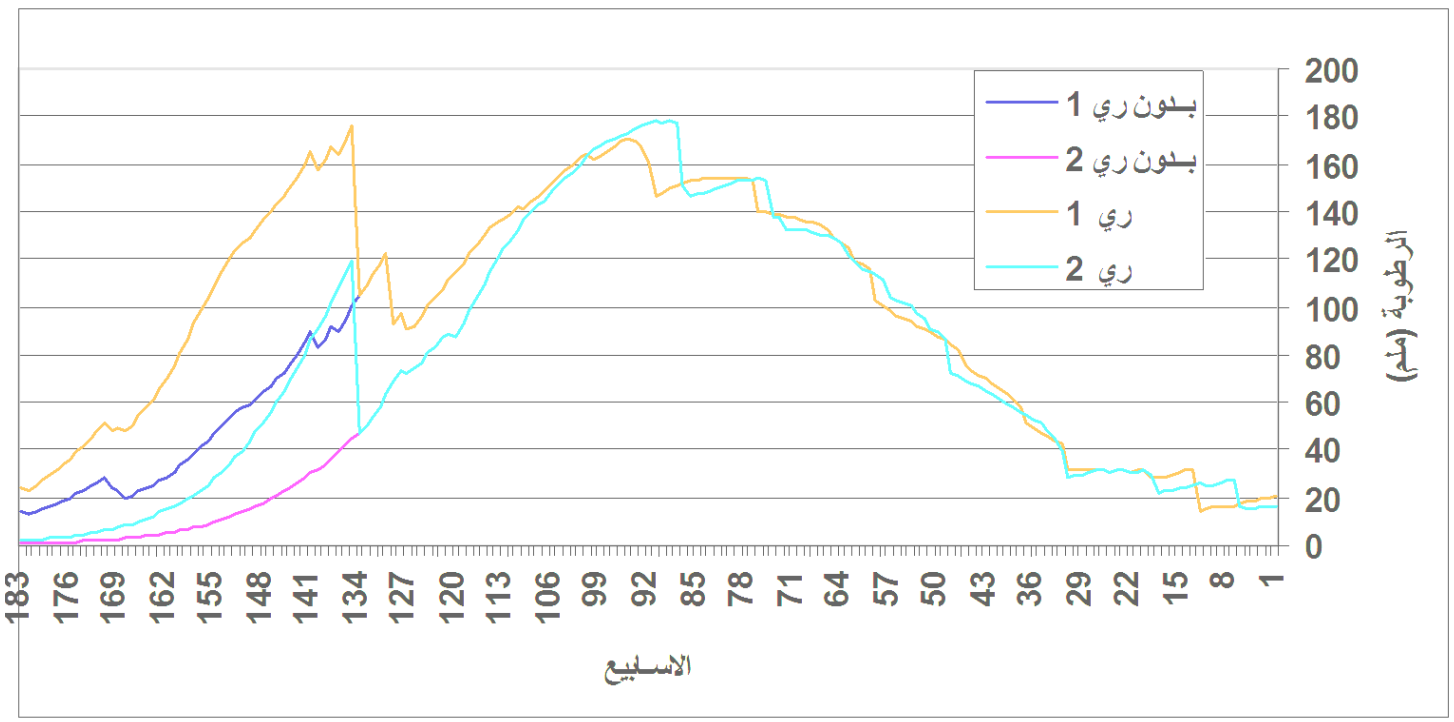

الثكل(7):التوزيع الرطوبي لكل اسبوع من الاسابيع على طول موسم النمو لسنة 1980-1981 


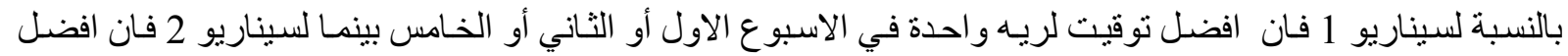

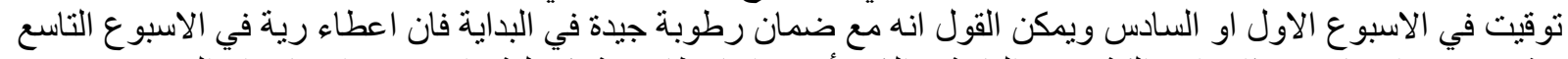

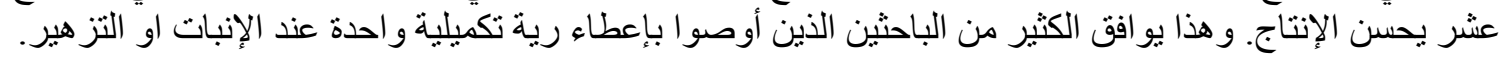

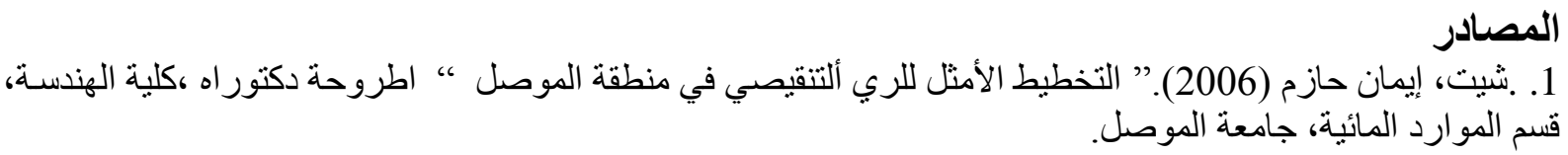

2. Allen, R.G. , Pereira, L.S., Raes ,D. and Smith ,M. (1998). " Crop evapotranspirationGuidelines for computing crop water requirements " .FAO Irrigation and Drainage paper No.56, Rome, Italy.

3. Awchi, T. A. (1990). "Dual scheduling of linear move irrigation system in AL-Jezera project ". M.Sc. thesis, College of Engineering, University of Mosul, Iraq, pp. 128.

4. Borg, H. and D.W.Grimes (1986). "Depth development of roots with time: An empirical description ".Trans. ASAE, Vol. 29(1), 194-197.

5. Doorenbos, J. and Kassam A. H, (1979). "Yield response to water ". FAO Irrigation and Drainage Paper No. 33.

6. Farahani,H. ,T.Oweis, H.Siadad, F.Abbasi, A.Bruggeman, J.Anthofer and F. Turkeloom. (2007)." Improving Water Productivity and Livelihood Resilience in Karkheh RiverBasin". Proceeding of the international workshop, sept 10-11,007, Karaj,Iran.

7.Oweis .Th , Ryan . J , and Pala .M , (1998) "Stabilizing rain fed wheat yields with supplemental irrigation and nitrogen in a Mediterranean climate " reprinted from agronomy journal Vol. 90, No .5 .

8.Tadayon,M.R. and Y.Emam. (2008)."Effect of Supplemental Irrigation and amount of available water on yield, yield components and physiological characteristics of Two Rain fed Wheat Cultivators" J.Sci and Technol. Agric and Natur. Resour. ,vol. 11, no.42 (A), Iran.

9.Sarvestani Z.T., S.A.M. Sanavy and A.Roohi (2004)." Yield and Yield Components of Dry Land Wheat Genotypes Under Supplemental Irrigation". Proceeding for the $4^{\text {th }}$ international crop science congress. Tarbiat Modarras University, Iran.

10 Tavakoli, Alireza; Zad Hassan, (2005)" Determining Optimal Single Irrigation Amount and Planting Date, Dry Land Agricultureal Reseach Institute, Maragheh (Iran),

URL: http://www.agrisis.org 